\title{
Influence of Introversion and Extraversion Using MBTI Personality Model on Academic Performance
}

\author{
Xuechao Li, Rodrigo Sardinas, Po-Chou Shih, and Karl Camp
}

\begin{abstract}
To date, research work in exploring the influence of personality on performance in programming courses has often been neglected due to the difficulty of quantitatively defining and measuring human factors. In this paper an empirical experiment was conducted in: (1) measuring Introversion/Extraversion personality types based on Myers-Briggs Type Indicator(MBTI); (2) defining scores of students' assignments as academic performance; and (3) using statistical analysis to verify our hypotheses about the linear relationship between personality types and academic performance. With the support of Independent T-test analysis, conclusions were found that introversion programmers outperformed extroversion programmers in their respective programming courses. In addition, the Pearson Correlation and regression analysis were utilized in order to assist educators in conducting more effective teaching styles in their programming courses.
\end{abstract}

Index Terms-Academic performance, empirical study, extraversion, introversion, Myers-Briggs type indicator.

\section{INTRODUCTION}

From serial coding to high performance computing [1]-[3], from social media to commercial applications such as vehicle tag recognition [4], technological advancements have played an important part in shaping the course of humanity. With this in mind it is now more crucial than ever to train up skillful programmers for the future. Until now educational institutions have attributed academic success in their programming courses to students' overall dedication and cognitive abilities overlooking personality as not relevant to an individual's success in their respective classes. Therefore, this paper seeks to build upon the current research on the subject matter and apply it in the area of academics.

This paper, then, contributes to the research in this field by conducting and analyzing an empirical study to find relationships between Introversion/Extraversion personality types and academic performance. Further, this paper presents a guideline for computer science educators to help them effectively advise students with different personality types in their programming courses. Finally, a regression model was presented which may be used to predict a student's academic performance based on their personality type. Our conclusions

Manuscript received November 15, 2017; revised April 24, 2018.

Xuechao Li is with the Concordia University Chicago, River Forest, IL 60305 USA (e-mail: Xuechao.Li@cuchicago.edu).

Rodrigo Sardinas is with the Auburn University, Auburn AL 36849 USA (e-mail: ras0054@tigermail.auburn.edu).

Po-Chou Shih is with National Taipei University of Technology, 10608 Taiwan.

Karl Camp is with Concordia University Chicago, River Forest, IL 60305 USA (e-mail: crf_campkl@ cuchicago.edu). supported our hypotheses in that introverted students generally outperformed extroverted students in terms of assignment scores in their respective programming courses.

The remainder of this paper is organized as follows: within literature review (section II) the various research is discussed that exists in the realms of education, psychology, and computer science on the topic of impact of various personality types on academic performance. In our main research (Section III) they were discussed that our motivation and hypotheses for conducting the empirical study and the methodologies used to perform the experiment. In research analysis (Section IV) the results are reviewed that were conducted in our experiment from independent T-tests, Pearson Correlation, and regression analysis. Finally, in section $\mathrm{V}$ our conclusions and future work to improve research in the field were presented.

\section{LITERATURE REVIEW}

From the establishment of formal education systems philosophers, educators, and researchers have explored various methodologies in properly educating the next generation of leaders [5]. With the development of computers and relevant computer science curriculum, the education of programmers has also come to the forefront of topics discussed within computer science. Over the past century, educators have studied various techniques to improve institutional curriculums in order to reflect the IT industries demanding needs. In addition, researchers have dedicated years of exploring correlations between programming performance and human factors. Since various definitions of performance and types of human factors lead to different conclusions, in this section the literature on the research that exists in the field were discussed and reviewed.

\section{A. Discussion on Personality Tests}

Research within the field of human factors and personality has come to light with the development of personality tests that accurately capture the inner world of those being tested. Currently there exist two personality models that are largely used to conduct research: the Big Five-Factor model (FFM) and the MBTI [6]. The FFM explores human behaviors, such as openness, conscientiousness, etc. in order to describe an individual's personality. The MBTI, on the other hand, looks at an individual's cognitive preferences, such as Introversion, Thinking, etc. exploring their personality in terms of unconscious choices a person makes in their daily activities. The MBTI model was selected, since statistical analysis has proven that the MBTI tends to be more accurate over time than the FFM [7]. Further, the FFM does not explore the 
nature of programming decisions effectively, since programming styles and choices are often dictated by unconscious coding preferences of the programmer.

\section{B. Personality and Academic Performance}

The idea that certain personality attributes impact academic performance has been researched since the turn of the last century. Chamorro-Premuzic and Furnham [8] were early researchers on the relationship between personality and academic performance. The authors compared two British University samples over a three year period and used several indicators, such as absenteeism, essay writing, and tutors' exam predictions, such as in predicting academic performance. In their research they utilized the FFM model proposed by Costa \& McCrae [9], specifically focusing on Neuroticism and Conscientiousness personality types to predict the overall final grade of students. They concluded that Neuroticism may impair academic performance, while Conscientiousness may lead to higher academic achievement Similar research was conducted by Hazrati et al. [10] in their study of personality and academic motivation, concluding that conscientiousness predicted intrinsic and extrinsic motivation, but openness to experience predicted only intrinsic behavior. Both studies were important in their attempt to find relationships between personality and academic performance.

\section{MBTI and Academic Performance}

MBTI has also been used in research within related topics. For example, the MBTI model was used in Ciorbea and Pasarica's [11] to research the relationship between personality and academic performance. The model was used to identify the level of organization during academic activities, particularly looking at Judging and Perceiving personality types. They also used Extended Project Qualification (EPQ) to measure Neuroticism, Extraversion and Psychoticism. In their study they concluded that (1) Neuroticism negatively influences academic performance; (2) Extraversion is only partially related to academic performance; and (3) Psychoticism is negatively associated with academic performance. Further, the students with Judgment personality type outperformed students with Perception personality type.

Though the use of MBTI model is more appropriate in the study of the relationship between academic performance and personality, our research improves upon previous research by focusing on the Extraversion and Introversion personality types in our study.

\section{Personality and Programming Courses}

Research in the field of human factors and academic performance has also been conducted within Computer Science. Golding et al. [12] researched the effects of peer tutoring, attitude and personality on academic performance among first year computer science students. The experiment was conducted at the University of Technology, Jamaica, during the fall semester of 2005, incorporating the MBTI personality traits as their identification of different personalities. The results showed little correlation between personality and academic performance. This correlated with Bishop-Clark's [13] research on the MBTI personalities and their relationship on different stages of programming. Given our focus on extroversion and introversion, our research challenges the above claims.

\section{MAIN RESEARCH}

Computer technologies have been developed for decades and training skillful programmers is becoming critical in an ever developing and expanding IT field. In general, educators provide projects, assignments, and exams in hopes that their students will improve their programming skills. Further, these assignments often try to imitate what an average programmer could face during a real project to prepare the students for a future career in software engineering. To this end, educators spend hours improving their courses to meet the industry's needs and cater to the students' ability to learn new programming concepts. However, the impact of human factors on a student's academic performance is often ignored. Hence, it is proposed that an empirical experiment explores the influence of students' personality on academic performance: specifically Introversion and Extraversion.

\section{A. Research Hypothesis}

The MBTI describes people as either being extraverted or introverted. Extraverted people prefer interacting with people outside of themselves, finding energy through socialization and constant engagement with their peers. Introverted people, on the other hand, prefer to spend time alone participating in activities they enjoy, often finding interaction with people draining. We suspected that extraverted programmers would exhibit deteriorated code performance, since the discussion and inclusion of various ideas would lead to inconsistent programming styles. Hence, it was hypothesized that introverted programmers perform better on programming assignments as compared to their extraverted peers. In regards to programmers with introversion types it was also hypothesized that they would exhibit programming consistency and, as a result, better code performance. Therefore, the following hypotheses were proposed:

H1: Programmers with Introversion characteristic can achieve better academic performance than programmers with Extraversion characteristic.

$\mathrm{H} 2$ : Introversion and Extraversion types can be linearly dependent on the academic performance.

H3: A regression model can be presented to estimate the expectation of academic performance influenced by Introversion.

\section{B. Data Collection}

Participants in the study were students enrolled in COMP 1200 at Auburn University, during the spring semester of 2017. Out of the 421 students, 324 voluntarily participated in the research. Academic performance was measured through seven project assignments during the semester. The personality survey was posted on SurveyMonkey website to be accessed by students at their own convenience.

Our participants' demographics are described in Table I. Since this experiment was conducted in an introduction level course, over $70 \%$ students' ages were 18 or 19 and $80 \%$ students are male and $20 \%$ students are female. Also, it is 
important to point out that about three quarters of the students had less than six months of programming experience, and most had never written code exceeding 1000 lines of code (LOC). It should also be noted that the overall academic achievement of the participants was a B+ level.

The MBTI personality survey was utilized in order to collect the personality type of each student. Ten questions were used from the survey, each including a specific statement as regards the individual [6]. The individual then decided whether or not they agreed with the statement being a reflection of themselves and answer in categories ranging from 1 (Disagree) to 5 (Agree). The survey then used Keirsey temperament sorter [14] to assess the student's personality type based on the answers.

\section{Project Description}

Academic performance was defined in terms of scores throughout each project. There were in total seven projects, each of which was divided among four assignments. The first assignment incorporated two simple mathematical problems exploring the understanding of basic arithmetic and trigonometry. Those were used to assess basic logic skills and allow for the student to become familiar with using MatLab. The second assignment tasks the student to improve the bubble sort algorithm based pseudo- code provided. This task would display an individual's ability to follow instructions and critically think about algorithm efficiency. The third assignment asked to use matrixes and perform basic mathematic operations, such as describing the mean, mode, etc, assessing the students understanding and manipulation of basic data structures. The final assignment was the most challenging, consisted of three projects, all of which used file processing in order to properly conduct complex algebraic functions. These projects covered a variety of programming topics so that measurement of program performance would be accurate for out empirical study.

\section{RESEARCH ANALYSIS}

In this section our statistical approach is described to support our claims and the results of our empirical study. The independent T-test was utilized to find correlations between personality and academic performance, the Pearson correlation to determine whether or not the variables are linearly dependent on each other, and regression analysis in order to predict the effect of personality academic performance.

TABLE I: PARTICIPANTS' DEMOGRAPHICS

\begin{tabular}{l|l|l}
\multicolumn{2}{c}{ TABLE I: PARTICIPANTS' DEMOGRAPHICS } \\
\hline \hline Variable & Item & $\begin{array}{l}\text { Number of } \\
\text { Participants }\end{array}$ \\
\hline \multirow{4}{*}{ Age } & 17 & 2 \\
& 18 & 118 \\
& 19 & 113 \\
& 20 & 54 \\
& 21 & 22 \\
& 22 & 7 \\
& 23 & 4 \\
& 2 & 1 \\
\hline \hline
\end{tabular}

\begin{tabular}{l|l|l}
\hline \hline Gender & Male & 257 \\
& Female & 64 \\
& 0 month & 6 \\
Year of Programming & 0 month-6 months & 241 \\
Experience & 6 month-12 months & 16 \\
& $>12$ months & 43 \\
& C/C++ & 37 \\
Programming & Java & 31 \\
Language & C\# & 4 \\
& Python & 15 \\
Largest & CUDA & 1 \\
Contribution & C100 LOC & 190 \\
Academic & 100 LOC - 1000 LOC & 122 \\
Achievement & 1000 LOC - 5000 LOC & 12 \\
\hline \hline
\end{tabular}

\section{A. Independent T-test}

To prove that programmers with Introversion characteristic can achieve a better grade than programmers with Extraversion characteristic, the independent T-test [15] was used. It is statistically accepted that p-values of less than 0.05 is a sign of significant difference for the comparisons. Statistically, if the p-values is less than 0.05 , it is reasonably believed that there is a significant difference for the comparison. Through the methodology described it was able to prove our hypotheses.

Since each assignment consisted of different problems, students who correctly completed all tasks within an individual assignment were considered in this experiment. For example, in the fourth assignment only 172 introverted students and 69 extraverted students solved the problems correctly.

Although the actual number of students who were able to successfully complete each of the assigned tasks varied in the study, some trends were clear: since all p-values were less than 0.05 across all assignments in Table II, it is reasonably made that the conclusion that introverted students performed better than their extroverted classmates. Taking Assignment 1 in Table 2 as an example, Introversion students were scored 91.70, while Extraversion students were scored 88.34. Since the p-value is less than 0.05 , statistically Introversion students outperformed Extraversion students.

An additional explanation was added behind our conclusions. Since extraverted students enjoy discussing questions with others, programming habits and styles were less consistent compared to the coding styles of introverted students. Hence, introverted students significantly outperformed extraverted students on the project assignments.

TABLE II: IMPACT OF PERSONALITY ON ACADEMIC PERFORMANCE

\begin{tabular}{|c|c|c|c|c|}
\hline Assignment & $\begin{array}{c}\text { Index } \\
\text { Extraversion }\end{array}$ & \multicolumn{2}{|c|}{ Grade } & P-value \\
\hline \multirow{2}{*}{1} & Extraversion & $\begin{array}{l}\text { Mean } \\
\text { Std. } \\
\mathrm{n}=182\end{array}$ & $\begin{array}{l}88.34, \\
15.13,\end{array}$ & \multirow{2}{*}{0.0193} \\
\hline & Introversion & Mean & 91.70 & \\
\hline
\end{tabular}




\begin{tabular}{|c|c|c|c|c|}
\hline & & $\begin{array}{l}\text { Std. } \\
\mathrm{n}=83\end{array}$ & 10.53 , & \\
\hline \multirow{2}{*}{2} & Extraversion & $\begin{array}{l}\text { Mean } \\
\text { Std. } \\
\mathrm{n}=184\end{array}$ & $\begin{array}{l}79.11, \\
22.35,\end{array}$ & \multirow{2}{*}{0.017} \\
\hline & Introversion & $\begin{array}{l}\text { Mean } \\
\text { Std. } \\
\mathrm{n}=82\end{array}$ & $\begin{array}{l}84.18, \\
15.50,\end{array}$ & \\
\hline \multirow{2}{*}{3} & Extraversion & $\begin{array}{l}\text { Mean } \\
\text { Std. } \\
\mathrm{n}=171\end{array}$ & $\begin{array}{l}83.09, \\
22.51,\end{array}$ & \multirow{2}{*}{0.037} \\
\hline & Introversion & $\begin{array}{l}\text { Mean } \\
\text { Std. } \\
\mathrm{n}=74\end{array}$ & $\begin{array}{l}87.77, \\
16.81,\end{array}$ & \\
\hline \multirow{2}{*}{4} & Extraversion & $\begin{array}{l}\text { Mean } \\
\text { Std. } \\
\mathrm{n}=172\end{array}$ & $\begin{array}{l}83.27, \\
20.15,\end{array}$ & \multirow{2}{*}{0.0025} \\
\hline & Introversion & $\begin{array}{l}\text { Mean } \\
\text { Std. } \\
n=69\end{array}$ & $\begin{array}{l}89.85, \\
14.39,\end{array}$ & \\
\hline
\end{tabular}

\section{B. Pearson Correlation}

This experiment used the Pearson correlation analysis [16] to explore whether or not a linear relationship exists between academic performance and personality. Statistically, if the absolute value of the coefficient is greater than 0.7 , it is believed that a linear relationship exists.

As seen in Table III, there is a noticeable strong linear dependence between students' grades and their Introversion character in project 2 and project 3 since the absolute values of coefficient value were greater than 0.7 . It means that students' academic performance is linearly dependent on introversion rather than extraversion.

Because we created a simple assignment 1 as a warm-up practice and a complicated assignment 4 to challenge the students, both assignments cannot be used to distinguish students' programming skills. According to this consideration, both assignments were took out as corner cases in the statistical analysis. Hence, a conclusion is made that the linear relationship exists between academic performance and personality.

\section{Regression Analysis}

The regression analysis was utilized, with R-square of 0.7 , in order to determine whether our prediction was convincing [17]. For each student, there is a corresponding value $\alpha$ for

\begin{tabular}{l|l}
\multicolumn{2}{c}{ TABLE III: CORRELATION ANALYSIS } \\
\hline \hline Assignment & Extraversion vs. Introversion \\
\hline 1 & -0.05496 \\
2 & -0.75081 \\
3 & -0.72657 \\
4 & -0.12858 \\
\hline \hline
\end{tabular}

\begin{tabular}{|c|c|c|c|c|c|}
\hline \multicolumn{6}{|c|}{ TABLE IV: REGRESSION ANALYSIS } \\
\hline Project & & Coefficient & P-val. & R2 & $\mathrm{N}$ \\
\hline \multirow[b]{2}{*}{ Grade } & Intercept & 0.51 & $8.37 \mathrm{E}-41$ & \multirow{3}{*}{0.67} & \multirow{3}{*}{208} \\
\hline & $\mathrm{EI}^{\prime}$ & 0.0048 & $4.58 \mathrm{E}-05$ & & \\
\hline $\begin{array}{l}\text { Regression } \\
\text { Equation }\end{array}$ & \multicolumn{3}{|c|}{$0.0048 * \mathrm{E}+0.51$} & & \\
\hline
\end{tabular}

Extraversion and $\beta$ for Introversion. It was converted that the "Introversion" value $\beta$ to the "Extraversion" value $\alpha$ in order to calculate the coefficient and the intercept in the regression equation. This conversion was done by: (1) calculating the maximum value $\alpha$ for five questions in the Introversion type; and (2) calculating the difference $d$ between the maximum value and the actual value from students. Since the maximum value for each question is 5 , the maximum value $\gamma$ for the Introversion type is 25 . The value of $d$ was then added to the value $\alpha$ for the original Extraversion type. We did a conversion from the Introversion to Extraversion, so the performance is linearly dependent on the Extraversion type if the coefficient is a positive number. The performance is linearly dependent on the Introversion type if the coefficient is a negative number. Since it is able to show that there exists a strong linear relationship between personality and academic performance, naturally it is explored whether it was possible to use the regression analysis to predict performance based on personality. In Table IV, the R-value is greater than 0.7 , but the residual plots also needed to be evaluated to make sure that all points are evenly distributed above and below the $\mathrm{x}$-axis.

As seen in Fig. 1, although some points are below or above the horizontal axis, overall all points are still randomly dispersed around the x-axis. It is concluded that our model was valid to predict the academic performance based on personality.

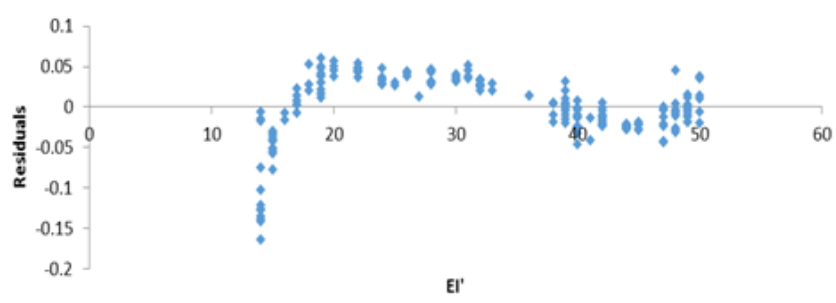

Fig 1. Extraversion vs. introversion residual plot.

\section{Threats to Validity}

For each step of the analysis we took an objective approach in conducting each trial in order to find correlations between academic performance and human personality. Due to the difficulty of such a task there is a potential for inaccuracy in our conclusions.

1) The complexity of the project must be taken into consideration. It is vital to the success of the experiment to choose problems that are solvable by the participants. However, if the tasks are solved by everyone or not solved by anyone, then our data would be skewed showing that correlations do not exist between personality and academic performance. Though we tried our best to choose problems that were solvable and span across all programming practices, this challenge still allows us to question our conclusions.

2) The academic level of participants also plays a vital role in the accuracy of our data. More experienced programmers can solve challenges quicker than students who have never done coding prior to college. Therefore, the academic level of our participants also calls our conclusions into question.

3) It is difficult to measure academic performance based 
solely on projects. Though it is believed that programming projects are an accurate representation of one's coding ability, academics does not simply consist of projects. Exams, homework assignments, work, family obligations, etc. all play an important factor in students' academic performance. Therefore, analyzing a broader spectrum of variables affecting academic performance might also impact our conclusions.

\section{CONCLUSIONS AND FUTURE WORK}

In our empirical study we explored: (1) whether there exists a correlation between academic performance and the personality; (2) whether there exists a significant academic difference between Introversion and Extraversion; and (3) whether a correlation can be mathematically predicted. Our conclusion shows that there does exist a correlation and that academic performance can be predicted based on a student's personality preference. Our empirical experiment was conducted through the use of independent T-tests, Pearson correlation, and regression models in order to provide an objective view on human personality. For example, if the Pearson correlation evaluation showed a value greater than 0.7 it was safe to conclude that a correlation existed between variables.

Future work includes exploring how the rest of the MBTI personality types affect academic performance, which will be analyzed through similar statistical analysis. Further, it can recruit more experienced programmers to solve the same problems to see if there are the same correlations which would either support our claims or call us to revise our conclusions. Finally, our solutions will then be run on computers with different configurations using more test cases to further analyze and predict correlations between variables.

\section{REFERENCES}

[1] M. Abid, "HPC (high-performance the computing) for big data on cloud: Opportunities and challenges," International Journal of Computer Theory and Engineering, vol. 8, no. 5, pp. 423-428, 2016.

[2] X. Li, P.-C. Shih, J. Overbey, C. Seals, and A. Lim, "Comparing programmer productivity in OpenACC AND CUDA: An empirical investigation," International Journal of Computer Science, Engineering and Application (IJCSEA), vol. 6, no. 5, October 2016.

[3] X. Li, P.-C. Shih, X.-Q. Li, and C. Seals, "A case study of novice programmers on parallel programming models," Journal of Computers, vol. 13, no. 5, May 2017.

[4] X. Li, C. Li, and Y. Xie, "A retrieval system of vehicles based on recognition of license plates," in Proc. 2011 International Conference on Machine Learning and Cybernetics (ICMLC), IEEE, Guilin, pp. 1453-1459, July 10-13, 2011.
[5] M. Farahani, M. Mirzamohamadi, and N. Noroozi, "The study on features of informal education in postmodernism," Procedia - Social and Behavioral Sciences, vol. 136, pp. 559-563, 2014.

[6] The Myers and Briggs Foundation. [Online]. Available: http://www.myersbriggs.org.

[7] X. Li, "The influence of human factors on programming performance: Personality, programming styles and programming attitudes," Ph.D. dissertation, Dept. Comp Sci \& Software Eng, Auburn University, Auburn, AL, 2017

[8] T. C. Premuzic and A. Furnham, "Personality predicts academic performance: Evidence from two longitudinal university samples," Journal of Research in Personality, vol. 37, no. 4, pp. 319-338, 2003.

[9] P. T. Jr. Costa and R. R. McCrae, "NEO PI-R professional manual Revised NEO PI-R and NEO-FFI," Florida, FL: Psychological Assessment Resources, Inc. 1992.

[10] A. Hazrati-Viari, A. T. Rad, and S. S. Torabi, "The effect of personality traits on academic performance: The mediating role of academic motivation," Procedia - Social and Behavioral Sciences, vol. 32, pp 367-371, 2012

[11] I. Ciorbea and F. Pasarica, "The study of the relationship between personality and academic performance," Procedia - Social and Behavioral Sciences, vol. 78, pp. 400-404, 2013.

[12] P. Golding, L. Facey-Shaw, and V. Tennant, "Effects of peer tutoring, attitude and personality on academic performance of first year introductory programming students," in Proc. the Frontiers in Education Conference, pp. 7-12. 2006.

[13] C. B. Clark, "Cognitive style and its effect on the stages of programming," Journal of Research on Computing Education, vol. 27 pp. 373-386. 1995.

[14] Keirsey temperament sorter. [Online]. Available: http://www.thechangeworkscoaching.com/images/Kiersey_Answer_S Shee_Sample_Ans_Sheet_Extra_Ans_Sheet_Scoring_Instructions_Fo Fo.pdf

[15] D. Howell, "Pacific grove: Duxbury," Statistical Methods for Psychology, 2002.

[16] J. Benesty, J. Chen, Y. Huang, and I. Cohen, "Pearson correlation coefficient," Noise Reduction in Speech Processing, pp 1-4, Springer Topics in Signal Processing, 2009.

[17] R. Freund, W. Wilson, and P. Sa, "Regression analysis," Academic Press, 2006

Xuechao Li received the Ph.D degree in Computer Science and Software Engineering Department at Auburn University USA 2017. He is an assistant professor at Concordia University Chicago and research interests include parallel computing, higher performance compiler optimization and empirical software engineering.

Rodrigo Sardinas is a $\mathrm{Ph} . \mathrm{D}$ candidate in computer science and software engineering department at Auburn University USA. His research work focuses on cybersecurity and high performance computing.

Po-Chou Shih is a Ph.D candidate in graduate institute of industrial and business management at National Taipei University of Technology, Taiwan. His research area is optimization of artificial intelligence technologies.

Karl Camp is a junior student at Concordia University Chicago. His work is to apply artificial intelligence technologies into optimizations of OpenACC compiler. 